\title{
A New Generalization of Negative Ploya- Eggenberger Distribution and its Applications
}

Anwar Hassan

University of Kashmir

Sheikh Nilal Ahmad

Degree College, Baramulla

Follow this and additional works at: http://digitalcommons.wayne.edu/jmasm

Part of the Applied Statistics Commons, Social and Behavioral Sciences Commons, and the Statistical Theory Commons

\section{Recommended Citation}

Hassan, Anwar and Ahmad, Sheikh Nilal (2007) "A New Generalization of Negative Ploya-Eggenberger Distribution and its Applications," Journal of Modern Applied Statistical Methods: Vol. 6 : Iss. 2 , Article 30.

DOI: $10.22237 /$ jmasm/1193891340

Available at: http://digitalcommons.wayne.edu/jmasm/vol6/iss2/30 


\section{A New Generalization of Negative Polya-Eggenberger Distribution and its Applications}

\author{
Anwar Hassan \\ University of Kashmir
}

\author{
Sheikh Bilal Ahmad \\ Degree College, Baramulla
}

A new generalization of negative Polya-Eggenberger distribution (GNPED) has been obtained by mixing the negative binomial distribution with generalized beta distribution- $\Pi$ defined by Nadarajah and Kotz (2003). Some special cases and properties of GNPED have been studied. Further, the proposed model has been fitted to two data sets (used by Gupta \& Ong, 2004) that provide a satisfactory fit and better alternative as compared to negative binomial and some of its mixture models and extensions. Also, the negative Polya-Eggenberger distribution (NPED), obtained by mixing negative binomial with beta distribution of I-kind, has been fitted to the same data sets for comparison.

Key words: negative binomial distribution, generalized beta distribution- $\Pi$, generalized negative PolyaEggenberger distribution (GNPED).

\section{Introduction}

Feller (1943) pointed out there are essentially two kinds of contagious distributions. One type, true contagion, is due to the fact that each favorable event increases (or decreases) the probability of succeeding favorable events. The other type, apparent contagion, is due to an inhomogeneity of the population. Frequently, the data arising in studies of entomology and bacteriology cannot be described by the usual distribution functions but rather by some type of contagious distributions. Some distributions, such as the negative binomial, can apparently be interpreted on the basis of both types of contagion.

A class of contagious distributions is derived from a certain biological model which takes into account the fact that the distribution of larvae over the plots of a field depends upon the

Anwar Hassan is a Post Graduate, Department. of Statistics. Research interests: Probability and Lagrangian Probability distributions and Statistical Inference. E-mail address: anwar.hassan2007@gmail.com. Sheikh Bilal Ahmad, Department of Statistics, E-mail: sbilal_sbilal@yahoo.com Research interests: Probability distributions and Statistical Inference. fact that the larvae are hatched from egg-masses which appear at random over the field has been derived by Neyman (1939), Evans (1953) and Beal and Rescia (1953). This class of distribution has been successful in accounting for the distribution of some insect populations (ef. Beal-1940). Bliss and Fisher (1953) showed that the negative binomial distribution is useful as a possible underlying distribution for insect populations. Contagious distributions have also been used in the study of accident and medical statistics by Dubourdieu (1939), Greenwood and Yule (1920), Lundberg (1940) and Newbold (1927). Eggenberger and Polya (1923) introduced Polya-Eggenberger distribution (PED) and negative Polya-Eggenberger distribution (NPED) by an urn model and described these as truly contagious distributions.

Negative Polya-Eggenberger Distribution (NPED)

Negative Polya-Eggenberger distribution is related to Polya-Eggenberger distribution in the same way as the negative binomial distribution is related to binomial distribution. It is well known fact that the negative binomial distribution (NBD) has become increasingly popular as a more flexible alternative to the Poisson distribution especially when it is doubtful whether the strict requirements 
particularly independence for a Poisson distribution will be statisfied. Negative PolyaEggenberger distribution is obtained by mixing negative binomial distribution

$$
\begin{gathered}
P(X=x)=\left(\begin{array}{c}
n+x-1 \\
x
\end{array}\right) p^{x}(1-p)^{n}, \\
x=0,1,2, \ldots ; o<p<1
\end{gathered}
$$

With beta distribution of I-Kind

$$
\begin{gathered}
P(X=p)=\frac{1}{\beta(\alpha, \gamma)} \quad p^{\alpha-1} \\
(1-p)^{\gamma-1} \\
0<p<1 \\
\alpha, \gamma>0
\end{gathered}
$$

If the parameter $p$ in (1) is not a constant but is varying as beta distribution of I-kind then its probability mass function is (2) and beta mixture of negative binomial distribution is obtained as

$$
\begin{gathered}
P(X=x)= \\
\left(\begin{array}{c}
n+x-1 \\
x
\end{array}\right) \frac{1}{\beta(\alpha, \gamma)} \int_{0}^{1} p^{x+\alpha-1} \\
(1-p)^{n+\gamma-1} d p \\
x=0,1,2, \ldots . . \\
=\left(\begin{array}{c}
n+x-1 \\
x
\end{array}\right) \frac{\beta(\alpha+x, \gamma+n)}{\beta(\alpha, \gamma)} \\
=\left(\begin{array}{c}
n+x-1 \\
x
\end{array}\right) \frac{(\alpha+\gamma-1) !}{(\alpha-1) !(\gamma-1) !} \\
\frac{(\alpha+x-1) !(\gamma+n-1) !}{(\alpha+\gamma+n+x-1) !} \\
P(X=x)= \\
\frac{\left(\begin{array}{c}
n+x-1 \\
x
\end{array}\right)}{(\alpha+1) \ldots \ldots\left(\alpha+\frac{1}{x-1}\right) \gamma(\gamma+1) \ldots \ldots . .(\gamma+\overline{n-1})}
\end{gathered}
$$

Taking $\alpha=a / c$ and $\gamma=b / c$ in the equation above, we get

$$
\begin{gathered}
P(X=x)= \\
\left(\begin{array}{c}
n+x-1 \\
x
\end{array}\right) \\
\frac{a(a+c) \ldots \ldots .(a+\overline{x-1} c) b(b+c) \ldots \ldots \ldots(b+\overline{n-1} c)}{(a+b)(a+b+c) \ldots \ldots \ldots . .(a+b+\overline{n+x-1} c)} \\
x=0,1,2, \ldots \ldots .
\end{gathered}
$$

Which is negative Polya-Eggenberger distribution with parameters (n, a, b, c).

Generalized Beta Distribution-II

Many generalization of beta distribution of I-kind (2) involving algebraic and exponential function has been proposed in the literature; see chapter 25 in Johnson et al (1995) and Gupta Nadarajah (2004) for detailed accounts. Nadarajah and Kotz (2003) defined a generalization of (2) involving the Gauss hypergeometric function as

$$
\begin{gathered}
P(X=p)= \\
\frac{b \beta(a, b)}{\beta(a, b+\gamma)} p_{2}^{a+b-1}{ }_{2} F_{1}[1-\gamma, a ; a+b, p],
\end{gathered}
$$

This is known as generalized beta distribution-II. The properties of incomplete beta function and Gauss hypergeometric function can be found in Prudnikov et al (1990, vol.3 sec. 7.3) and Gradshteyn and Ryzhik (2000).

There are various extensions/ modifications of NBD in the literature including Engen's extended NBD (1974, 1978), generalized NBD of Jain and Consul (1971) and weighted NBD; see Johnson et al. (1992) for more details and explanations. A brief list of authors and their work can be seen in Johnson and Kotz (1969), Consul and Famoye (2000), Johnson and Balkrishnan (1995) and Gupta and Nadarajah (2004).

In this article, an attempt has been made to introduce a new generalized contagious distribution, generalized negative PolyaEggenberger distribution (GNPED), by mixing NBD with generalized beta distribution- $\Pi$ defined by Nadarajah and Kotz's (2003) which is expected to explain data in a better way as compared to distributions obtained by mixing Poison or binomial with other distributions. Further, the proposed model has been fitted to 
same data sets previously used by Gupta and Ong (2004) that exhibits a satisfactory fit and better alternative as compared to negative binomial, negative Polya-Eggenberger, Gupta and Ong's (2004) GNBD and Jain and Consul's (1971) GNBD.

The Proposed Model

Let $\mathrm{X}$ be a random variable representing the number of independent trails necessary to obtain ' $n$ ' occurrences of an event that has a constant probability of occurring at each trail. Then $\mathrm{X}$ has a negative binomial distribution with parameters (n, p) and pmf given by (1). But, there are situations in the practical life where probability ' $p$ ' of each occurrence of an event is not constant but is following some distribution. In the present case, suppose ' $p$ ' is varying as generalized beta distribution- $\Pi$ by Nadarajah and Kotz (2003) with parameters $(a, b, \gamma)$ and pdf given by (4) then the pmf of proposed model is obtained by mixing (1) with (4) as

$$
\begin{gathered}
P(X=x)=\left(\begin{array}{c}
x+n-1 \\
n-1
\end{array}\right) \frac{b \beta(a, b)}{\beta(a, b+\gamma)} \\
\int_{0}^{1}\left[p^{n+a+b-1}(1-p)^{x}\right. \\
{ }_{2} F_{1}[1-\gamma, a ; a+b, p] d p \\
=\left(\begin{array}{c}
x+n-1 \\
n-1
\end{array}\right) \frac{b \beta(a, b)}{\beta(a, b+\gamma)} \\
\sum_{j=0}^{\infty}\left[\frac{(1-\gamma)^{[j]} a^{[j]}}{(a+b)^{[j]}} \frac{1}{j !}\right. \\
\left.\int_{0}^{1}\left[p^{n+a+b+j-1}(1-p)^{x}\right] d p\right]
\end{gathered}
$$

Using Gradibhtyn and Ryzhik's (2000) book, we obtain after few steps

$$
\begin{gathered}
P(X=x)= \\
\frac{(x+n-1) !}{(n-1) ! \quad \frac{b \beta(a, b)}{\beta(a, b+\gamma)} \frac{(a+b)^{[n]}}{(a+b)^{[n+x+1]}}} \\
\times{ }_{3} F_{2}[1-\gamma, a, a+b+n ; a+b, a+b+n+x+1,1] \\
x=0,1,2 \ldots
\end{gathered}
$$

This is a probability mass function of the proposed model, generalized negative PolyaEggenberger distribution (GNPED), which can further be simplified to give

$$
\begin{gathered}
P(X=x)= \\
\frac{(x+n-1) !}{(n-1) !} \frac{(a+b)^{[\gamma]}(a+b)^{[n]}}{(b+1)^{[\gamma-1]}(a+b)^{[n+x+1]}} \\
{ }_{3} F_{2}[1-\gamma, a, a+b+n ; a+b, a+b+n+x+1,1] \\
x=0,1, \ldots \ldots \ldots . .
\end{gathered}
$$

Some Special Cases of GNPED

Some old and new distributions can be obtained by assigning different values to the parameters of GNPED (6).

For $a+b+\gamma=1,(6)$ reduces to

$$
\begin{gathered}
P(X=x)= \\
\frac{(x+n-1) !}{(n-1) !} \frac{(a+b)^{[\gamma]}(a+b)^{[n]}}{(b+1)^{[\gamma-1]}(a+b)^{[n+x+1]}} \\
{ }_{2} F_{1}[a, a+b+n ; a+b+n+x+1,1] \\
x=0,1, \ldots \ldots \ldots .
\end{gathered}
$$

The Gauss summation theorem states that

$$
{ }_{2} F_{1}[a, b ; c, 1]=\frac{\Gamma c \Gamma(c-a-b)}{\Gamma(c-a) \Gamma(c-b)}
$$

Provided $(c-a-b)>0, c \neq 0,-1,-2,-3, \ldots \ldots$

Using (8) in (7), we obtain NPED with pmf

$$
\begin{gathered}
P(X=x)= \\
\left(\begin{array}{c}
x+n-1 \\
n-1
\end{array}\right)
\end{gathered}
$$

$\frac{(1-a)(1-a+1) \ldots . .(1-a+\overline{x-1})(a+b)(a+b+1) \ldots(a+b+\overline{n-1})}{(1+b)(1+b+1) \ldots \ldots \ldots .(1+b+\overline{n+x-1})}$

If one puts $(1-a)=\frac{\alpha}{c} \&(a+b)=\frac{\beta}{c}$ $\Rightarrow(1+b)=\frac{(\alpha+\beta)}{c}$, then the above equation reduces to NPED in its usual form as

$$
P(X=x)=
$$




$$
\begin{gathered}
\left(\begin{array}{c}
x+n-1 \\
n-1
\end{array}\right) \\
\frac{\alpha(\alpha+c) \ldots \ldots .(\alpha+\overline{x-1} c) \beta(\beta+c) \ldots \ldots \ldots .(\beta+\overline{n-1} c)}{(\alpha+\beta)(\alpha+\beta+c) \ldots \ldots \ldots(\alpha+\beta+\overline{n+x-1} c)} \\
x=0,1, \ldots \ldots
\end{gathered}
$$

For $\gamma=1,(6)$ reduces to

$$
\begin{gathered}
P(X=x)= \\
\frac{(a+b)}{(a+b+n+x)} \\
\frac{\beta(a+b, x+n)}{\beta(a+b, n)} \\
x=0,1, \ldots \ldots \ldots . .
\end{gathered}
$$

Where ${ }_{3} F_{2}[1-\gamma, a, a+b+n ; a+b$,

$a+b+n+x+1,1]=1$ for $\gamma=1$

If in addition, one puts $\mathrm{a}+\mathrm{b}=1$, then (11) reduces to factorial distribution

$$
\begin{gathered}
P(X=x)= \\
\frac{n}{(n+x)(n+x+1)} \\
x=0,1,2, \ldots .
\end{gathered}
$$

For $b=0,(6)$ reduces to

$$
\begin{gathered}
P(X=x)= \\
\frac{(n+x-1) !}{(n-1) !} \frac{a^{[\gamma]} a^{[n]}}{1^{[\gamma-1]} a^{[n+x+1]}} \\
{ }_{2} F_{1}[1-\gamma, a+n ; a+n+x+1,1]
\end{gathered}
$$

Using Gauss summation theorem (8), we obtain on simplifications

$$
\begin{gathered}
P(X=x)= \\
\left(\begin{array}{c}
x+n-1 \\
n-1
\end{array}\right) \frac{a^{[n]} \gamma^{[x]}}{(a+\gamma)^{[n+x]}} \\
x=0,1, \ldots \ldots .
\end{gathered}
$$

If one puts $\gamma=\frac{\alpha}{c} \& a=\frac{\beta}{c}$, then above equation reduces to negative Polya-Eggenberger distribution (9).
For $\mathrm{a}=0,(6)$ reduces to

$$
\begin{gathered}
P(X=x)= \\
\frac{b}{(b+n+x)} \frac{\beta(b, n+x)}{\beta(b, n)} \\
x=0,1,2, \ldots .
\end{gathered}
$$

Where,

$$
{ }_{3} F_{2}[1-\gamma, a, a+b+n ; a+b, a+b+n+x+1,1]=1,
$$

for $a=0$. If in addition one puts $b=1$, then (13) reduces to (12).

$$
\text { For }(a+b)=1,(6) \text { reduces to }
$$

$$
\begin{gathered}
P(X=x)= \\
\frac{n}{(n+x)(n+x+1)} \frac{b ! \gamma !}{(b+\gamma-1) !} \\
{ }_{3} F_{2}[1-\gamma, a, 1+n ; 1, n+x+2,1]
\end{gathered}
$$

If in addition one puts

i) $\mathrm{a}=0$, then (14) reduces to

$$
P(X=x)=\frac{n b \gamma \beta(b, \gamma)}{(x+x)(x+n+1)}
$$

This is a new generalization of factorial distribution (12) as it reduces to (12) for $\gamma=1$ or $b=1$

If one replaces $\mathrm{x}$ with $(\mathrm{x}-\mathrm{n})$, then (6) reduces to

$$
\begin{gathered}
P(X=x)= \\
\frac{(x-1) !}{(n-1) !} \frac{b \beta(a, b)}{\beta(a, b+\gamma)} \frac{(a+b)^{[n]}}{(a+b)^{[x+1]}} \\
{ }_{3} F_{2}[1-\gamma, a, a+b+n ; a+b, a+b+x+1,1]
\end{gathered}
$$

Taking $\gamma=1$ and $b=0$, this results in a new distribution with pmf

$$
\begin{gathered}
P(X=x)= \\
\frac{n a}{x}\left(\begin{array}{c}
x \\
x-n
\end{array}\right)\left[\left(\begin{array}{c}
a+x \\
x-n
\end{array}\right)\right]^{-1} \\
x=n, n+1, n+2, \ldots \ldots .
\end{gathered}
$$


Moment Generating Function of GNPED

Moment generating function of (5) can be obtained as

$$
\begin{gathered}
M_{X}(t)=E\left(e^{t X}\right) \\
=\sum_{x=0}^{\infty} e^{t x} \frac{(x+n-1) !}{(n-1) !} \frac{b \beta(a, b)}{\beta(a, b+\gamma)} \frac{(a+b)^{[n]}}{(a+b)^{[n+x+1]}} \\
{ }_{3} F_{2}[1-\gamma, a, a+b+n ; a+b, a+b+x+1,1] \\
=\frac{b \beta(a, b)}{\beta(a, b+\gamma)} \\
\sum_{j=0}^{\infty} \frac{(1-\gamma)^{[j]} a^{[j]}(a+b+n)^{[j]}}{(a+b)^{[j]}} \frac{1}{j !} \\
\times \sum_{x=0}^{\infty} \frac{n^{[x]} 1^{[x]}}{\frac{\left(e^{t}\right)^{x}}{x !}}
\end{gathered}
$$

On simplification, this gives moment generating function of GNPED as

$$
\begin{gathered}
M_{X}(t)= \\
\frac{b \beta(a, b)}{\beta(a, \gamma+b)} \sum_{j=0}^{\infty} \frac{(1-\gamma)^{[j]} a^{[j]}(n+a+b)^{[j]}}{(a+b)^{[j]}(n+a+b)^{[j+1]}} \\
\times \frac{{ }_{2} F_{1}\left[n, 1 ; n+a+b+j+1, e^{t}\right]}{j !}
\end{gathered}
$$

Remarks:

If one replaces $e^{t}$ with $(1-t)^{-1}$ in (15), ascending factorial moment generating function is obtained as

$$
\begin{gathered}
E(1-t)^{-X}= \\
\frac{b \beta(a, b)}{(a+b+n) \beta(a, b+\gamma)} \\
\sum_{j=0}^{\infty} \frac{(1-\gamma)^{[j]} a^{[j]}(a+b+n)^{[j]}}{(a+b)^{[j]}(a+b+n+1)^{[j]}} \frac{1}{j !} \\
\times{ }_{2} F_{1}\left[n, 1 ; a+b+n+j+1,(1-t)^{-1}\right]
\end{gathered}
$$

Similarly, replacing $e^{t}$ with $(1+t)$ in (15), descending factorial moment generating function of GNPED is obtained as

$$
\begin{gathered}
E(1+t)^{X}= \\
\frac{b \beta(a, b)}{(a+b+n) \beta(a, b+\gamma)} \\
\sum_{j=0}^{\infty} \frac{(1-\gamma)^{[j]} a^{[j]}(a+b+n)^{[j]}}{(a+b)^{[j]}(a+b+n+1)^{[j]}} \frac{1}{j !} \\
\times_{2} F_{1}\left[n, 1 ; a+b+n+j+1, e^{t}\right]
\end{gathered}
$$

\section{Raw Moments of GNPED}

The $\mathrm{r}^{\text {th }}$ raw moment of the proposed model (5) can be obtained as

$$
E\left(X^{r}\right)=E\left[E\left(X^{r} / p\right)\right]
$$

Where $E\left(X^{r} / p\right)$ is the conditional $\mathrm{rth}$ moment of $\mathrm{X}$ for given $p$ and for given $p$, the random variable $X$ has negative binomial distribution (1) with

$$
E\left(X^{r} / p\right)=\sum_{x=1}^{r}\left(\begin{array}{c}
x+n-1 \\
n-1
\end{array}\right) p^{-x}(1-p)^{x} \Delta^{x} 0^{r}
$$

Hence, (16) reduces to

$$
E\left(X^{r)}=\sum_{x=1}^{r}\left(\begin{array}{c}
x+n-1 \\
n-1
\end{array}\right) E\left[p^{-x}(1-p)^{x}\right] \Delta^{x} 0^{r}\right.
$$

Because $p$ is varying as generalized beta distribution$\Pi$ (2) with parameters $(a, b, \gamma)$. Therefore,

$$
\begin{gathered}
\mu_{r}^{\prime}= \\
\frac{b \beta(a, b)}{\beta(a, b+\gamma)} \sum_{x=1}^{r}\left(\begin{array}{c}
x+n-1 \\
n-1
\end{array}\right) \Delta^{x} 0^{r} \\
\int_{0}^{1} p^{a+b-x-1}(1-p)^{1+x-1} \\
{ }_{2} F_{1}[1-\gamma, a ; a+b, p] d p
\end{gathered}
$$

This on simplification gives the rth moment of GNPED as

$$
\begin{gathered}
\mu_{r}^{\prime}=\frac{b \beta(a, b)}{\beta(a, b+\gamma)} \\
\sum_{x=1}^{r} \frac{(x+n-1) !(a+b-x-1) !}{(n-1) !(a+b) !} \Delta^{x} 0^{r}
\end{gathered}
$$




$$
\times_{3} F_{2}[1-\gamma, a, a+b-x ; a+b, a+b+1,1]
$$

Taking $r=1,2,3,4$ in (17), one gets first four raw moments as

$$
\begin{aligned}
& \mu_{1}^{\prime}=\frac{n b \beta(a, b)}{(a+b)(a+b-1) \beta(a, b+\gamma)} \\
& { }_{3} F_{2}[1-\gamma, a, a+b-1 ; a+b, a+b+1,1] \\
& \mu_{2}^{\prime}=\frac{n b \beta(a, b)}{(a+b)(a+b-1) \beta(a, b+\gamma)} \\
& { }_{3} F_{2}[1-\gamma, a, a+b-1 ; a+b, a+b+1,1] \\
& +\frac{2 n(n+1) b \beta(a, b)}{(a+b)(a+b-1)(a+b-2) \beta(a, b+\gamma)} \\
& { }_{3} F_{2}[1-\gamma, a, a+b-2 ; a+b, a+b+1,1] \\
& \mu_{3}^{\prime}=\frac{n b \beta(a, b)}{(a+b)(a+b-1) \beta(a, b+\gamma)} \\
& { }_{3} F_{2}[1-\gamma, a, a+b-1 ; a+b, a+b+1,1] \\
& +\frac{6 n(n+1) b \beta(a, b)}{(a+b)(a+b-1)(a+b-2) \beta(a, b+\gamma)} \\
& { }_{3} F_{2}[1-\gamma, a, a+b-2 ; a+b, a+b+1,1] \\
& +\frac{6 n(n+1)(n+2) b \beta(a, b)}{(a+b)(a+b-1)(a+b-2)(a+b-3) \beta(a, b+\gamma)} \\
& \times{ }_{3} F_{2}[1-\gamma, a, a+b-3 ; a+b, a+b+1,1] \\
& \mu_{4}^{\prime}=\frac{n b \beta(a, b)}{(a+b)(a+b-1) \beta(a, b+\gamma)} \\
& { }_{3} F_{2}[1-\gamma, a, a+b-1 ; a+b, a+b+1,1] \\
& +\frac{14 n(n+1) b \beta(a, b)}{(a+b)(a+b-1)(a+b-2) \beta(a, b+\gamma)} \\
& { }_{3} F_{2}[1-\gamma, a, a+b-2 ; a+b, a+b+1,1] \\
& +\frac{36 n(n+1)(n+2) b \beta(a, b)}{(a+b)(a+b-1)(a+b-2)(a+b-3) \beta(a, b+\gamma)} \\
& \times_{3} F_{2}[1-\gamma, a, a+b-3 ; a+b, a+b+1,1] \\
& +\frac{24 n(n+1)(n+2)(n+3) b \beta(a, b)}{(a+b)(a+b-1)(a+b-2)(a+b-3)(a+b-4) \beta(a, b+\gamma)} \\
& \times{ }_{3} F_{2}[1-\gamma, a, a+b-4 ; a+b, a+b+1,1]
\end{aligned}
$$

Descending Factorial Moments of GNPED
The $\mathrm{r}^{\text {th }}$ descending factorial moment of (5) can be obtained as

$$
E\left(X^{(r)}\right)=E\left[E\left(X^{(r)} / p\right)\right]
$$

Where for given $\mathrm{p}$, the random variable ' $\mathrm{X}$ ' follows negative binomial distribution with

$$
E\left(X^{(r)} / p\right)=(n+r-1)^{(r)} p^{-r}(1-p)^{r}
$$

Proceeding in the same way as above, the $r^{\text {th }}$ descending factorial moment of GNPED is

$$
\begin{gathered}
\mu_{(r)}^{\prime}=\frac{(n+r-1)^{(r)}(a+b-r-1) r ! b \beta(a, b)}{(a+b) ! \beta(a, b+\gamma)} \\
\times_{3} F_{2}[1-\gamma, a, a+b-r ; a+b, a+b+1,1]
\end{gathered}
$$

Taking $\mathrm{r}=1,2,3,4$ in (18), one gets first four factorial moments as

$$
\begin{gathered}
\mu_{(1)}^{\prime}=\frac{n b \beta(a, b)}{(a+b)(a+b-1) \beta(a, b+\gamma)} \\
{ }_{3} F_{2}[1-\gamma, a, a+b-1 ; a+b, a+b+1,1] \\
\mu_{(2)}^{\prime}=\frac{2 n(n+1) b \beta(a, b)}{(a+b)(a+b-1)(a+b-2) \beta(a, b+\gamma)} \\
{ }_{3} F_{2}[1-\gamma, a, a+b-2 ; a+b, a+b+1,1] \\
\mu_{(3)}^{\prime}=\frac{6 n(n+1)(n+2) b \beta(a, b)}{(a+b)(a+b-1)(a+b-2)(a+b-3) \beta(a, b+\gamma)} \\
\times{ }_{3} F_{2}[1-\gamma, a, a+b-3 ; a+b, a+b+1,1] \\
\frac{\mu_{(4)}^{\prime}=}{(a+b)(a+b-1)(a+b-2)(a+b-3)(a+b-4) \beta(a, b+\gamma)} \\
\times{ }_{3} F_{2}[1-\gamma, a, a+b-4 ; a+b, a+b+1,1]
\end{gathered}
$$

Central Moments of GNPED

$$
\begin{gathered}
\mu_{2}=\frac{n b \beta(a, b)}{\beta(a, \gamma+b)(a+b)(a+b-1)} \\
{ }_{3} F_{2}[1-\gamma, a, a+b-1 ; a+b, a+b+1,1]
\end{gathered}
$$




$$
\begin{aligned}
& +\frac{2 n(n+1) b \beta(a, b)}{\beta(a, \gamma+b)(a+b)(a+b-1)(a+b-2)} \\
& { }_{3} F_{2}[1-\gamma, a, a+b-2 ; a+b, a+b+1,1] \\
& -\left[\frac{n b \beta(a, b)}{\beta(a, \gamma+b)(a+b)(a+b-1)}{ }_{3} F_{2}[1-\gamma, a, a+b-1 ; a+b, a+b+1,1]\right]^{2} \\
& \mu_{3}=\frac{n b \beta(a, b)}{\beta(a, \gamma+b)(a+b)(a+b-1)} \\
& { }_{3} F_{2}[1-\gamma, a, a+b-1 ; a+b, a+b+1,1] \\
& -3\left\{\frac{n b \beta(a, b)}{\beta(a, \gamma+b)(a+b)(a+b-1)}{ }_{3} F_{2}[1-\gamma, a, a+b-1 ; a+b, a+b+1,1]\right\}^{2} \\
& +2\left\{\frac{n b \beta(a, b)}{\beta(a, \gamma+b)(a+b)(a+b-1)}{ }_{3} F_{2}[1-\gamma, a, a+b-1 ; a+b, a+b+1,1]\right\}^{3} \\
& +\frac{6 n(n+1) b \beta(a, b)}{\beta(a, \gamma+b)(a+b)(a+b-1)(a+b-2)} \\
& { }_{3} F_{2}[1-\gamma, a, a+b-2 ; a+b, a+b+1,1] \\
& \times\left\{1-\frac{n b \beta(a, b)}{\beta(a, \gamma+b)(a+b)(a+b-1)}{ }_{3} F_{2}[1-\gamma, a, a+b-1 ; a+b, a+b+1,1]\right\} \\
& +\frac{6(n+1)(n+2) b \beta(a, b)}{\beta(a, \gamma+b)(a+b)(a+b-1)(a+b-2)(a+b-3)} \\
& \times{ }_{3} F_{2}[1-\gamma, a, a+b-3 ; a+b, a+b+1,1] \\
& \mu_{4}=\frac{n b \beta(a, b)}{\beta(a, \gamma+b)(a+b)(a+b-1)} \\
& { }_{3} F_{2}[1-\gamma, a, a+b-1 ; a+b, a+b+1,1] \\
& +\frac{2 n(n+1) b \beta(a, b)}{\beta(a, \gamma+b)(a+b)(a+b-1)(a+b-2)} \\
& { }_{3} F_{2}[1-\gamma, a, a+b-2 ; a+b, a+b+1,1] \\
& \times\left[7-\frac{12 n b \beta(a, b)}{\beta(a, \gamma+b)(a+b)(a+b-1)}\right. \\
& { }_{3} F_{2}[1-\gamma, a, a+b-1 ; a+b, a+b+1,1] \\
& \left.+6\left\{\frac{n b \beta(a, b)}{\beta(a, \gamma+b)(a+b)(a+b-1)}{ }_{3} F_{2}[1-\gamma, a, a+b-1 ; a+b, a+b+1,1]\right\}^{2}\right] \\
& +\frac{12 n(n+1)(n+2) b \beta(a, b)}{\beta(a, \gamma+b)(a+b)(a+b-1)(a+b-2)(a+b-3)} \\
& \times{ }_{3} F_{2}[1-\gamma, a, a+b-3 ; a+b, a+b+1,1] \\
& \times\left[3-\frac{2 n b \beta(a, b)}{\beta(a, \gamma+b)(a+b)(a+b-1)}\right. \\
& \left.{ }_{3} F_{2}[1-\gamma, a, a+b-1 ; a+b, a+b+1,1]\right]
\end{aligned}
$$

$$
\begin{aligned}
& +\frac{24(n+1)(n+2)(n+3) b \beta(a, b)}{\beta(a, \gamma+b)(a+b)(a+b-1)(a+b-2)(a+b-3)(a+b-4)} \\
& \quad \times{ }_{3} F_{2}[1-\gamma, a, a+b-4 ; a+b, a+b+1,1] \\
& -4\left\{\frac{n b \beta(a, b)}{\beta(a, \gamma+b)(a+b)(a+b-1)}{ }_{3} F_{2}[1-\gamma, a, a+b-1 ; a+b, a+b+1,1]\right\}^{2} \\
& +6\left\{\frac{n b \beta(a, b)}{\beta(a, \gamma+b)(a+b)(a+b-1)}{ }_{3} F_{2}[1-\gamma, a, a+b-1 ; a+b, a+b+1,1]\right\}^{3} \\
& -3\left\{\frac{n b \beta(a, b)}{\beta(a, \gamma+b)(a+b)(a+b-1)}{ }_{3} F_{2}[1-\gamma, a, a+b-1 ; a+b, a+b+1,1]\right\}^{4}
\end{aligned}
$$

Goodness of Fit

Gupta and Ong (2004) obtained GNBD by mixing NBD with generalized gamma distribution defined by Amero and Bayrr (1933) and Agarwal and Kalla (1996). The pmf of GNBD is

$$
\begin{gathered}
P(X=x)= \\
\left(\begin{array}{c}
m+x-1 \\
x
\end{array}\right)\left(\frac{\alpha}{1+\alpha}\right)^{m-\lambda}\left(\frac{1}{1+\alpha}\right)^{x} \\
\times \frac{\phi(\lambda, \lambda-m+1-x ;(\alpha+1) n)}{\phi(\lambda, \lambda-m+1 ; \alpha n)}
\end{gathered}
$$

Where $(m, \alpha, \lambda, n)$ are the parameters of the distribution and $\phi(\lambda, \lambda-m+1-x ;(\alpha+1) n)$ is a confluent hypergeometric function.

Gupta and Ong demonstrated the goodness of fit test for their model (19) with the help of two data sets [Tables (1)-(2)] and observed marked fist than NBD and Jain and Consul's (1971) GNBD.

In this section, the proposed model GNPED has also been fitted to these data sets to show that the proposed model exhibits the best fit as compared to other distributions such as NBD, Jain and Consul's (1971) GNBD and Gupta and Ong's (2004) GNBD. The negative Polya-Eggenberger distribution has also been fitted to these data sets for its comparison with these distributions. 
Table 1 Absenteeism among shift-workers in steel industry; data of Arbous and Sichel, 1954

\begin{tabular}{|c|c|c|c|c|c|c|}
\hline \multirow[t]{2}{*}{ Count } & \multirow{2}{*}{$\begin{array}{l}\text { Observed } \\
\text { Frequency }\end{array}$} & \multicolumn{5}{|c|}{ EXPECTED FREQUENCY } \\
\hline & & NBD & $\begin{array}{l}\text { Jain } r \text { and } \\
\text { Consul's (1971) } \\
\text { GNBD }\end{array}$ & $\begin{array}{l}\text { Ramesh } \\
\text { and Ong's } \\
(2004) \\
\text { GNBD }\end{array}$ & NPED & $\begin{array}{l}\text { PROPOSED } \\
\text { MODEL } \\
\text { GNPED }\end{array}$ \\
\hline 0 & 7 & 12.02 & 10.51 & 09.23 & 9.53 & 9.06 \\
\hline 1 & 16 & 16.16 & 17.45 & 16.18 & 15.93 & 16.79 \\
\hline 2 & 23 & 17.77 & 20.38 & 19.86 & 19.06 & 23.62 \\
\hline 3 & 20 & 18.08 & 20.80 & 21.06 & 19.92 & 22.89 \\
\hline 4 & 23 & 17.65 & 19.88 & 20.50 & 19.41 & 21.95 \\
\hline 5 & 24 & 16.80 & 18.34 & 18.78 & 18.17 & 20.67 \\
\hline 6 & 12 & 15.72 & 16.56 & 16.46 & 16.59 & 17.11 \\
\hline 7 & 13 & 14.52 & 14.78 & 14.02 & 14.90 & 15.24 \\
\hline 8 & 09 & 13.28 & 13.08 & 11.79 & 13.25 & 11.04 \\
\hline 9 & 09 & 12.06 & 11.53 & 09.95 & 11.71 & 8.78 \\
\hline 10 & 08 & 10.89 & 10.13 & 08.55 & 10.30 & 8.04 \\
\hline 11 & 10 & 09.78 & 08.89 & 07.54 & 9.04 & 7.21 \\
\hline 12 & 08 & 08.75 & 07.79 & 06.84 & 7.92 & 6.38 \\
\hline 13 & 07 & 07.80 & 16.83 & 06.33 & 6.94 & 5.82 \\
\hline 14 & 02 & 06.93 & 05.99 & 05.94 & 6.08 & 5.24 \\
\hline 15 & 12 & 06.14 & 05.26 & 05.61 & 5.33 & 4.73 \\
\hline 16 & 03 & 05.43 & 04.61 & 05.29 & 4.68 & 4.27 \\
\hline 17 & 05 & 04.79 & 04.05 & 04.97 & 4.12 & 3.96 \\
\hline 18 & 04 & 04.22 & 03.56 & 04.64 & 3.63 & 3.69 \\
\hline 19 & 02 & 03.17 & 03.14 & 04.28 & 3.20 & 3.46 \\
\hline 20 & 02 & 03.23 & 02.76 & 03.92 & 2.83 & 3.27 \\
\hline 21 & 05 & 02.86 & 02.43 & 03.55 & 2.50 & 2.98 \\
\hline 22 & 05 & 02.50 & 02.15 & 03.19 & 2.22 & 2.88 \\
\hline 23 & 02 & 02.91 & 01.90 & 02.84 & 1.97 & 2.67 \\
\hline 24 & 01 & 01.91 & 01.68 & 02.50 & 1.75 & 2.16 \\
\hline $25-48$ & 16 & 12.77 & 13.50 & 14.13 & 17.02 & 14.09 \\
\hline TOTAL & 248 & 248 & 248 & 248 & 248 & 248 \\
\hline \multirow[t]{6}{*}{ Estimates } & & $\mathrm{p}=0.854$ & $\alpha=0.00010775$ & $\lambda=0.6226$ & $\mathrm{n}=14.962954$ & $\mathrm{n}=100.09367$ \\
\hline & & $\mathrm{n}=1.576$ & $\beta=59785288$ & $\alpha=0.001$ & $\alpha=2.492821$ & $\gamma=1.00021$ \\
\hline & & & $\mathrm{n}=29337.0839$ & $\mathrm{~m}=0.1601$ & $\gamma=4.852530$ & $\mathrm{a}=2.24578$ \\
\hline & & & & $\mathrm{n}=0.01897$ & 10.20108 & \\
\hline & & $\begin{array}{l}\mathbf{1 4 . 9 2} \\
17\end{array}$ & 27.79 & $\begin{array}{l}8.27 \\
15\end{array}$ & 16 & $\begin{array}{l}7.621862 \\
15\end{array}$ \\
\hline & & & 17 & & & \\
\hline
\end{tabular}


Table 2 Counts of the number of European red mites on apple leaves; data of P.Garman, 1951

\begin{tabular}{|c|c|c|c|c|c|c|}
\hline Count & $\begin{array}{r}\text { Observed } \\
\text { Frequency }\end{array}$ & \multicolumn{5}{|c|}{ EXPECTED FREQUENCY } \\
\hline & & NBD & $\begin{array}{l}\text { Jain and } \\
\text { Consul(1971) } \\
\text { GNBD }\end{array}$ & \begin{tabular}{|l|} 
Ramesh \\
and Ong's \\
(2004) \\
GNBD
\end{tabular} & NPED & $\begin{array}{l}\text { PROPOSED } \\
\text { MODEL } \\
\text { GNPED }\end{array}$ \\
\hline $\begin{array}{l}0 \\
1 \\
2 \\
3 \\
4 \\
5 \\
6 \\
7 \\
8 \\
\end{array}$ & $\begin{array}{l}70 \\
38 \\
17 \\
10 \\
09 \\
03 \\
02 \\
01 \\
00 \\
\end{array}$ & $\begin{array}{l}69.49 \\
37.60 \\
20.10 \\
10.70 \\
05.69 \\
03.02 \\
01.60 \\
00.85 \\
00.95 \\
\end{array}$ & $\begin{array}{l}69.49 \\
37.60 \\
20.10 \\
10.70 \\
05.69 \\
3.02 \\
1.60 \\
0.85 \\
0.95 \\
\end{array}$ & \begin{tabular}{|l}
70.24 \\
37.05 \\
18.06 \\
11.03 \\
06.89 \\
03.79 \\
01.79 \\
00.74 \\
00.40 \\
\end{tabular} & $\begin{array}{r}69.19 \\
38.27 \\
20.09 \\
10.49 \\
6.51 \\
2.93 \\
1.57 \\
0.86 \\
0.09 \\
\end{array}$ & $\begin{array}{c}70.92 \\
36.87 \\
19.41 \\
10.34 \\
6.57 \\
3.04 \\
1.67 \\
0.93 \\
0.25 \\
\end{array}$ \\
\hline TOTAL & 150 & 150 & 150 & 150 & 150 & 150 \\
\hline $\begin{array}{l}\text { ML } \\
\text { Estimate } \\
\chi^{2} \\
\text { d.f }\end{array}$ & & $\begin{array}{l}\mathrm{p}=0.5281 \\
\mathrm{n}=1.0246 \\
\mathbf{2 . 4 8 4} \\
3\end{array}$ & $\begin{array}{l}\alpha=0.52810 \\
\beta=1.000 \\
m=1.0246 \\
\mathbf{2 . 4 8 4} \\
\mathbf{2}\end{array}$ & $\begin{array}{c}\lambda=65.170 \\
\alpha=1.73908 \\
\mathrm{~m}=66.6914 \\
\mathrm{n}=0.001 \\
\\
\quad \mathbf{0 . 9 3} \\
\mathbf{1}\end{array}$ & $\begin{array}{l}\mathrm{n}=22.924537 \\
\alpha=1.167381 \\
\gamma=24.297517 \\
\mathbf{1 . 5 1 7 4 4 3} \\
2\end{array}$ & $\begin{array}{l}n=37.72660 \\
\gamma=0.99869 \\
a=16.91825 \\
b=16.91825 \\
\\
1.257801 \\
1\end{array}$ \\
\hline
\end{tabular}

Note: The expected frequencies and the estimates for the parameters of the Jain and Consul's (1971) generalized negative binomial distribution are same at $\beta=1$ as given by negative binomial distribution shown in column third of the table (2).

The maximum likelihood estimate of the parameters of the proposed model have been obtained and shown at their respective places in the tables. It is mentioned here that due to complicated likelihood function, the ML estimates are determined by the same method as used by Gupta and Ong (2004) i.e. by a direct numerical search for global maximum of the log-likelihood surface. A random start procedure is employed i.e. for a set of random starting points this numerical search is repeated for each starting point in order to verify that the global maximum has been found.

It is evident from the tables 1 and 2 that the chi-square values of the proposed model (GNPED), in all the cases, gives the marked fit as compared to other distributions.

\section{References}

Beall, G. (1940). The fit and significance of contagious distributions when applied to observations on larval insects. Ecology, 21, 460474.

Beall G, \& Rescia, R.R. (1953).A generalization of Neyman's contagious distributions. Biometrics, 9, 354-57.

Bliss, C. I.,, Fisher, R. A. (1953). Fitting the negative binomial distribution to biological data and note on the efficient fitting of the negative binomial. Biometrics, 9, 176-200.

Bose, P.K. (1944). On confluent hyper geometric series. Sankhya, 6, 407-412. 
Dubourdieu, J. (1939) Theorie de l'assurance-maladie, Paris.

Eggenberger, F., \& Polya, G. (1923). Uber die Statistik verketteter vorgange. Z.Angew. Math. Mech.,1, 279-289.

Engen, S. (1974). On species frequency model. Biometrika, 61, 263-270

Engen, S. (1978). Stochastic abundance models. London: Chapman and Hall.

Evans, D. A. (1953). Experimental evidence concerning contagious distributions in ecology. Biometrika, 40,186-211.

Feller, W. (1943). On a general class of contagious distributions. Ann. Math. Stat., 14, 389-400.

Greenwood, M., \& Yule, G. U. (1920). An inquiry into the nature of frequency distribution representative of multiple happenings with particular reference to the occurrence of multiple attacks of disease or of repeated accidents. J. Roy. Stat. Soc., Ser. A 83, 255-279.
Jain G. C., \& Consul, P. C. (1971). A Generalized Negative Binomial Distribution. SIAM. J. Appl. Math, 21(.4.) 501-513

Johnson, N. L., \& Kotz, S (1969). Univariate discrete distribution. NY: John Wiley.

Lundberg, O. (1940). On random processes and their application to sickness and accident statistics. Thesis, University of Stockholm.

Nath, P. (1951) Confluent hyper geometric function. Sankhya, 11, 153-166.

Newbold, E. (1927). Practical applications of the statistics of repeated events, particularly to industrial accidents. J. Roy. Stat. Soc., 90, 487-547.

Neyman, J. (1939). On a new class of contagious distribution applicable in entomology and bacteriology. Ann. Math. Stat., 10, 35-57.

Slater, L.J (1953).On the evaluation of the confluent hyper geometric function. Proceedings of the Cambridge Philosophical Society, 49, 612-622. 